Article

\title{
Baseline Absolute Lymphocyte Count and ECOG Performance Score Are Associated with Survival in Advanced Non-Small Cell Lung Cancer Undergoing PD-1/PD-L1 Blockade
}

\author{
Florian Huemer ${ }^{1}$, David Lang ${ }^{2}$, Theresa Westphal ${ }^{1}$, Simon Peter Gampenrieder ${ }^{1}$, \\ Georg Hutarew ${ }^{3}$, Lukas Weiss ${ }^{1}$, Hubert Hack1 ${ }^{4}$ (D), Bernd Lamprecht ${ }^{2}$, Gabriel Rinnerthaler ${ }^{1,+}$ \\ and Richard Greil ${ }^{1, *,+}$ (D) \\ 1 Department of Internal Medicine III with Haematology, Medical Oncology, Haemostaseology, Infectiology \\ and Rheumatology, Oncologic Center, Salzburg Cancer Research Institute-Laboratory for Immunological \\ and Molecular Cancer Research (SCRI-LIMCR), Paracelsus Medical University, 5020 Salzburg, Austria \\ Department of Pulmonology, Kepler University Hospital, Med Campus III, 4020 Linz, Austria \\ Institute of Pathology, Paracelsus Medical University Salzburg, 5020 Salzburg, Austria \\ Division of Bioinformatics, Biocenter, Medical University of Innsbruck, 6020 Innsbruck, Austria \\ Correspondence: r.greil@salk.at \\ + These authors contributed equally to this work.
}

Received: 28 May 2019; Accepted: 8 July 2019; Published: 10 July 2019

\begin{abstract}
Immune-checkpoint blockade in front-line or second-line treatment improves survival in advanced non-small cell lung cancer (aNSCLC) when compared with chemotherapy alone. However, easily applicable predictive parameters are necessary to guide immune-checkpoint inhibition in clinical practice. In this retrospective bi-centric analysis, we investigated the impact of baseline patient and tumor characteristics on clinical outcome in aNSCLC patients treated with programmed cell death protein 1(PD-1)/programmed cell death ligand 1 (PD-L1) inhibitors. Between May 2015 and January 2018, 142 unselected consecutive NSCLC patients received PD-1/PD-L1 inhibitors during the course of disease. In multivariate analysis, we identified the Eastern Cooperative Oncology Group (ECOG) performance status (ECOG $>1$ versus ECOG $\leq 1$, HR: 3.23, 95\%CI: $1.58-6.60, P=0.001$ ), baseline absolute lymphocyte count (ALC; high: $>0.93 \times 10^{9} / \mathrm{L}$ versus low: $\leq 0.93 \times 10^{9} / \mathrm{L}, \mathrm{HR}: 0.38,95 \% \mathrm{CI}$ : $0.23-0.62, P<0.001$ ), prior or concomitant anti-vascular endothelial growth factor (VEGF) targeting therapy (yes versus no, HR: $2.18,95 \%$ CI: $1.15-4.14, P=0.017$ ) and TNM stage (IV versus III, HR: 4.18, $95 \%$ CI: 1.01-17.36, $P=0.049)$ as the most relevant parameters for survival. Neither antibiotic exposure (antibiotic-positive versus antibiotic-negative, HR: 0.90, 95\%CI: 0.56-1.45, $P=0.675$ ), nor PD-L1 expression on tumor cells ( $\geq 1 \%$ versus $<1 \%$, HR: $0.68,95 \%$ CI: $0.41-1.13, P=0.140$ ) was associated with survival. Baseline ECOG performance status and ALC were associated with survival in aNSCLC patients treated with PD-1/PD-L1 inhibitors and assessment of these parameters could be suitable in clinical practice.
\end{abstract}

Keywords: absolute lymphocyte count; ECOG performance status; immune-checkpoint inhibitor; antibiotics; PD-1/PD-L1; immune-checkpoint blockade; RANK; VEGF; denosumab

\section{Introduction}

Non-small cell lung cancer (NSCLC) represents the leading cause of cancer-mortality in the United States and in Europe [1,2]. The therapeutic concept of unleashing a pre-existing immune response against the tumor by the use of immune-checkpoint inhibitors results in long-term survival in $17 \%$ 
to $27 \%$ of patients with advanced NSCLC (aNSCLC) [3,4]. Meanwhile, positive phase III trial data support the use of nivolumab [5,6], pembrolizumab [7-10], atezolizumab [11,12], durvalumab [13], and ipilimumab combined with nivolumab [14] for systemic therapy in aNSCLC.

Despite achievement of overall response rates (ORR) between $45 \%$ to $48 \%$ by immune-checkpoint blockade (ICB) as monotherapy in programmed cell death ligand 1 (PD-L1) high expressing tumors or in combination with chemotherapy and despite a plateauing of overall survival (OS) curves after 12 to 15 months, half of the patients experience disease progression after 8.8 to 10.3 months from initiation of first-line therapy and will necessitate further systemic treatment [8,9]. Due to the rising costs caused by the therapeutic approach of ICB and, in order to select patients that will derive clinical benefit from currently approved immune-checkpoint inhibitor protocols, the identification of easily available predictive baseline parameters is an absolute necessity in the daily clinical practice. Several attempts have been made to predict the therapy response or treatment failure to ICB based on patient and tumor characteristics.

The PD-L1 expression on tumor cells has been extensively investigated as a predictive marker for ICB. Although the clinical outcome on immune-checkpoint inhibition in aNSCLC improves with higher PD-L1 expression [6,7,9-12,15], PD-L1 as a biomarker has several limitations. PD-L1 expression is heterogeneous and influenced by chemotherapy and targeted therapy [16]. Furthermore, staining differences among various antibody clones pose a challenge in clinical practice and PD-L1 negativity does not exclude a response to immune-checkpoint inhibition [17].

Results of a few recently published retrospective studies demonstrate a negative impact of antibiotic use in temporal proximity to the initiation of ICB for several tumor entities including NSCLC [18-22]. Fecal microbiota transplantation from cancer patients who responded to immune-checkpoint inhibitors into mice that had been pretreated with antibiotics restored responses to ICB in these animals [18]. The latter finding suggests a substantial role of the gut microbiota composition during immune-checkpoint inhibitor therapy.

Tumor mutational burden (TMB) represents another biomarker of interest to guide immune-checkpoint inhibitor therapy in various tumor entities [23]. Presentation of neoantigens generated by tumor somatic mutations is essential for tumor immunogenicity and the response to ICB $[24,25]$, whereas a TMB cut-off of $\geq 10$ mutations per mega base was clinically validated in NSCLC $[14,26]$.

Already established biomarkers such as driver mutations of the epidermal growth factor receptor (EGFR), which are generally sensitive to tyrosine-kinase inhibitors, also play a role when considering ICB since rising evidence suggests that single-agent immune-checkpoint inhibition is not active in NSCLC with sensitizing EGFR mutations $[6,27,28]$.

Inflammation is involved in the pathogenesis and promotion of cancer progression $[29,30]$ and is associated with worse clinical outcome in aNSCLC [31]. The suppression of lymphocyte and natural killer cell activity, the release of cytokines, which support tumor progression, and an adverse influence on the tumor microenvironment may contribute to a worse clinical outcome in a state of chronic inflammation [32]. Besides the C-reactive protein, the neutrophil-lymphocyte ratio (NLR) represents a marker of systemic inflammation, is of prognostic value in many solid tumors including NSCLC [32,33], and has also been proposed to predict the benefit from immune-checkpoint inhibition in aNSCLC [34-37].

Anti-vascular endothelial growth factor (VEGF) targeting therapy with the monoclonal antibody bevacizumab has been used in combination with chemotherapy in aNSCLC with non-squamous histology [38] and is recommended as front-line therapy in combination with chemotherapy and PD-L1 inhibition by the current NCCN guidelines in non-squamous histology aNSCLC [39]. Apart from inhibiting tumor angiogenesis, anti-VEGF targeting therapy exerts immunomodulatory effects [40] and, therefore, is an interesting combination partner for ICB.

Development of bone metastases during the course of aNSCLC is frequently observed. The monoclonal antibody denosumab inhibits osteoclast maturation [41], decreases skeletal-related 
events in patients with bone metastases from solid tumors [42], improves survival in aNSCLC with bone metastases [43], and is approved for the latter indication. Furthermore, denosumab acts as an immunomodulator [44,45], but its influence on ICB activity in vivo has to be clarified.

In this retrospective bicentric study, we report the impact of baseline patient characteristics, baseline laboratory parameters, and tumor characteristics on clinical outcome with programmed cell death protein 1 (PD-1)/PD-L1 inhibitors in a well-characterized advanced NSCLC cohort.

\section{Patients and Methods}

\subsection{Patients}

In this retrospective analysis, we included unselected consecutive patients with histologically confirmed aNSCLC (stage III/IV) that had been treated with immune-checkpoint inhibitors at the tertiary cancer centers in Salzburg (Austria) and Linz (Austria). Upon first presentation of positive phase III trial data for second-line aNSCLC [5-7] PD-1/PD-L1 inhibitors were applied as monotherapy within named patient programs, before the respective approval by the European Medicines Agency (EMA) and Food and Drug Administration (FDA). After drug approval and incorporation of immune-checkpoint inhibitors into the guidelines of the European Society of Medical Oncology (ESMO) and National Comprehensive Cancer Network (NCCN), these guidelines were followed. Patients receiving PD-1/PD-L1 inhibitors in combination with chemotherapy and anti-VEGF targeting therapy within clinical trials (clinicaltrials.gov identifier NCT02367794 and NCT02367794) were also included in this analysis.

\subsection{Data Collection}

Baseline patient characteristics and baseline laboratory values preceding the initiation of immune-checkpoint inhibitor therapy up to 14 days were retrospectively assessed. Data were extracted from medical records including age, sex, Eastern Cooperative Oncology Group (ECOG) performance status, TNM stage, histologic subtype, smoking history, EGFR mutation status, anaplastic lymphoma kinase (ALK) translocation status, central nervous systems (CNS) involvement, PD-L1 expression on tumor cells, immune-checkpoint inhibitor therapy line, immune-checkpoint inhibitor substance, prior or concomitant denosumab application, prior or concomitant anti-VEGF targeting therapy (bevacizumab, ramucirumab, and nintedanib), antibiotic treatment status, subsequent therapy protocols, absolute lymphocyte count (ALC), absolute neutrophil count (ANC), NLR (calculated as the ratio of the baseline ANC and ALC), C-reactive protein (CRP), and prior radiotherapy to the primary tumor or metastases.

Central assessment of the PD-L1 expression status for both oncologic centers was carried out by a single experienced lung pathologist. PD-L1 expression on tumor cells was assessed by immunohistochemistry utilizing the anti-PD-L1 clone 22C3 from Dako ${ }^{\circledR}$. In immune-checkpoint inhibitor clinical trials in aNSCLC a PD-L1 (tumor propensity score) cut-off value of $\geq 1 \%$ is frequently used for stratification and defined PD-L1 positivity in our analysis. Due to the low frequency of EGFR driver mutations among squamous NSCLC, testing for these oncogenic aberrations is not routinely carried out in our clinical practice and, therefore, these cases were considered as EGFR wild-type [46].

Radiologic reassessment by PET-CT or CT scan was performed every two to three months, or as clinically indicated. Progression-free survival (PFS) was calculated from the date of start of ICB until radiologically confirmed progression or death. Patients without progression at the last contact were censored. OS was calculated from the date of ICB initiation until death from any cause. Patients alive at the last contact were censored. Concomitant use of antibiotics was defined as the application within a time frame of one month before or one month after the initiation of ICB. 


\subsection{Statistical Analyses}

Differences in patient baseline characteristics between anti-VEGF therapy exposed and anti-VEGF naïve patients were tested by Pearson's $\chi^{2}$-test. For continuous data, the difference between the two groups was calculated with two-sided Wilcoxon rank-sum test. Maximal Harrell's C-index was used to find the optimal cut-off value for OS prediction for continuous data such as the ALC, ANC, and NLR (Table S1). In an exploratory analysis, we used the Kaplan-Meier method for survival curves and to evaluate PFS and OS differences according to baseline characteristics. Log-rank test was used to compare survival distributions between two patient groups. Median follow-up time was calculated using Kaplan-Meier curves where event indices (death versus censor) were switched. Kendall's tau coefficient (Kendall tau-b) was used to measure ordinal associations between parameters. Univariate Cox regression analyses were performed on OS and PFS for indicated, dichotomized, or binary patient data. Only significant variables in the univariate test $(P<0.05$, Wald test) were included in multivariate Cox regression models with an exception for NLR since it is directly dependent on ALC. We performed additional multivariate Cox regression analyses including clinically important parameters independent of significance (histology, age, and sex) and including all variables besides NLR and ANC. Proportional hazard assumptions were tested using the 'coxzph' function. All analyses were performed using the statistical software environment R (version 3.5.1, www.R-project.org, Vienna, Austria) including package 'survival.'

\section{Results}

Between May 2015 and January 2018, 142 patients with aNSCLC were treated with PD-1/PD-L1 inhibitors at the two tertiary cancer centers. At data cut-off (10 January 2018) after a median follow-up of 13.3 months, 109 patients had progressed on immune-checkpoint inhibitor therapy and 76 patients had died. The baseline characteristics are depicted in Table 1.

Table 1. Baseline characteristics of 142 advanced NSCLC patients.

\begin{tabular}{ccc}
\hline & $\mathbf{N}=\mathbf{1 4 2} \mathbf{( 1 0 0} \mathbf{0})$ & \\
\hline Age & Mean (Standard Deviation) & $66(10.6)$ \\
\hline \multirow{2}{*}{ Sex } & male & $85(60 \%)$ \\
& female & $57(40 \%)$ \\
\hline \multirow{2}{*}{ ECOG performance status } & 0 & $39(27 \%)$ \\
& 1 & $86(61 \%)$ \\
Histology & 2 & $14(10 \%)$ \\
& 3 & $3(2 \%)$ \\
\hline \multirow{2}{*}{ Smoking history } & non-squamous & $96(68 \%)$ \\
& squamous & $46(32 \%)$ \\
\hline \multirow{2}{*}{ TNM stage } & smoker & $116(88 \%)$ \\
& never-smoker & $16(12 \%)$ \\
& missing & $10(7 \%)$ \\
\hline ALK translocation & IIIA & $6(4 \%)$ \\
& IIIB & $8(6 \%)$ \\
& IIIC & $1(1 \%)$ \\
& IV & $127(89 \%)$ \\
\hline
\end{tabular}


Table 1. Cont.

\begin{tabular}{|c|c|c|}
\hline \multicolumn{3}{|c|}{$\mathrm{N}=142(100 \%)$} \\
\hline \multirow{2}{*}{ CNS involvement } & no & $112(79 \%)$ \\
\hline & yes & $30(21 \%)$ \\
\hline \multirow{3}{*}{ PD-L1 status } & positive & $75(63 \%)$ \\
\hline & negative & $44(37 \%)$ \\
\hline & missing & $23(16 \%)$ \\
\hline \multirow{3}{*}{ PD-L1 status category } & $<1 \%$ & $44(37 \%)$ \\
\hline & $1-50 \%$ & $39(33 \%)$ \\
\hline & $>50 \%$ & $35(30 \%)$ \\
\hline \multirow{3}{*}{ ICB therapy line } & 1st line & $40(28 \%)$ \\
\hline & 2nd line & $67(47 \%)$ \\
\hline & $\geq 3$ rd line & $35(25 \%)$ \\
\hline \multirow{3}{*}{ Immune-checkpoint inhibitor } & nivolumab & $79(55 \%)$ \\
\hline & pembrolizumab & $52(37 \%)$ \\
\hline & atezolizumab & $11(8 \%)$ \\
\hline \multirow{2}{*}{ Monotherapy versus combined therapy } & ICB monotherapy & $137(97 \%)$ \\
\hline & ICB combination therapy & $5(3 \%)$ \\
\hline \multirow{2}{*}{ Tertiary oncologic center } & Salzburg & $50(35 \%)$ \\
\hline & Linz & $92(65 \%)$ \\
\hline \multirow{2}{*}{ Prior/concomitant denosumab application } & no & $106(75 \%)$ \\
\hline & yes & $36(25 \%)$ \\
\hline \multirow{2}{*}{ Prior/concomitant anti-VEGF therapy * } & no & $125(88 \%)$ \\
\hline & yes & $17(12 \%)$ \\
\hline \multirow{2}{*}{ Prior radiotherapy \# } & no & $79(56 \%)$ \\
\hline & yes & $63(44 \%)$ \\
\hline \multirow{4}{*}{ Subsequent therapy } & no therapy & $85(60 \%)$ \\
\hline & taxane-based & $19(13 \%)$ \\
\hline & TKI & $17(12 \%)$ \\
\hline & other & $21(15 \%)$ \\
\hline \multirow{2}{*}{ Antibiotic treatment during ICB $\S$} & no & $80(56 \%)$ \\
\hline & yes & $62(44 \%)$ \\
\hline \multirow{7}{*}{ Antibiotic class } & penicillin & $45(73 \%)$ \\
\hline & fluoroquinolone & $27(44 \%)$ \\
\hline & cephalosporine & $12(19 \%)$ \\
\hline & carbapenem & $5(8 \%)$ \\
\hline & metronidazole & $5(8 \%)$ \\
\hline & macrolide & $4(6 \%)$ \\
\hline & linezolide & $2(3 \%)$ \\
\hline \multirow{7}{*}{ Antibiotic treatment indication } & empiric antibiotic therapy & $31(50 \%)$ \\
\hline & respiratory tract infection & $18(29 \%)$ \\
\hline & perioperative prophylaxis & $5(8 \%)$ \\
\hline & $\begin{array}{c}\text { gastrointestinal tract } \\
\text { infection }\end{array}$ & $4(6 \%)$ \\
\hline & biliary tract infection & $2(3 \%)$ \\
\hline & urinary tract infection & $1(2 \%)$ \\
\hline & $\begin{array}{l}\text { central venous catheter } \\
\text { infection }\end{array}$ & $1(2 \%)$ \\
\hline
\end{tabular}

ECOG: Eastern Cooperative Oncology Group, EGFR: epidermal growth factor receptor, ALK: anaplastic lymphoma kinase, CNS: central nervous system, PD-L1: programmed cell death ligand 1, ICB: immune-checkpoint blockade, VEGF: vascular endothelial growth factor, TKI: tyrosine kinase inhibitor. * bevacizumab, ramucirumab, or nintedanib. $\S$ administration of antibiotics within a time frame of one month before or one month after initiation of immune-checkpoint blockade. ${ }^{\#}$ to the primary tumor or metastases. 


\subsection{Progression-Free Survival}

Median PFS was 3.9 months (95\%CI: 3.1-5.7 months, Figure S1A).

In univariate analysis, the TNM stage (IV versus III, HR: $2.03,95 \% \mathrm{CI}: 1.02-4.03, P=0.044$ ), immune-checkpoint inhibitor therapy line ( $\geq 3$ rd line versus $<3$ rd line, HR: 1.73, 95\%CI: 1.13-2.63, $P=0.011$ ), PD-L1 expression status (positive versus negative, HR: $0.49,95 \% \mathrm{CI}: 0.32-0.75, P=0.001$, Figure S2A) (different cut-offs for PD-L1 $(<1 \%, 1-50 \%,>50 \%)$ resulted in a significant association with PFS, logrank $P=0.0038$ ), prior or concomitant application of anti-VEGF targeting therapy (yes versus no, HR: $1.89,95 \%$ CI: 1.07-3.36, $P=0.029$ ), ECOG performance status ( $>1$ versus $\leq 1$, HR: 2.53 , 95\%CI: 1.49-4.29, $P<0.001$ ) and ALC (high: $>0.93 \times 10^{9} / \mathrm{L}$ versus low: $\leq 0.93 \times 10^{9} / \mathrm{L}, \mathrm{HR}: 0.58,95 \% \mathrm{CI}$ : $0.38-0.88, P=0.010$ ) were statistically significantly associated with PFS. In multivariate analysis, only baseline ALC remained independently associated with PFS (HR: 0.55, 95\% CI: 0.35-0.88, $P=0.012$, Table 2A). ALC were still significantly associated with PFS for both if histology, age, and sex were included in the multivariate model $(P=0.030)$ and if all other variables besides NLR and ANC were included in a multivariate model $(P=0.023)$ (Table 2A).

\subsection{Overall Survival}

Median OS was 12.2 months (95\% CI: 10.7-15.1 months, Figure S1B). In univariate analysis, baseline NLR (high: $>3.8$ versus low: $\leq 3.8$, HR: 2.22, 95\%CI: 1.36-3.63, $P=0.001$, Figure 1A), TNM stage (IV versus III, HR: 5.52, 95\% CI: 1.35-22.64, $P=0.018$, Figure 2A), immune-checkpoint inhibitor therapy line ( $\geq 3$ rd line versus $<3$ rd line, HR: $1.97,95 \%$ CI: $1.24-3.16, P=0.004$ ), prior or concomitant application of anti-VEGF targeting therapy (yes versus no, HR: $2.18,95 \%$ CI: $1.21-3.93, P=0.010$, Figure 2B), ECOG performance status (ECOG $>1$ versus ECOG $\leq 1$, HR: 2.58, 95\% CI: 1.30-5.10, $P=0.007$, Figure $2 \mathrm{C}$ ), and ALC (high: $>0.93 \times 10^{9} / \mathrm{L}$ versus low: $\leq 0.93 \times 10^{9} / \mathrm{L}, \mathrm{HR}: 0.38,95 \% \mathrm{CI}$ : $0.24-0.62, P<0.001$, Figure 1B) showed a statistically significant association with OS (Table 2B). Due to the fact that differences in OS according to the NLR (Figure 1A) were mainly attributable to variations in the ALC (Figure 1B), but not to variations in the ANC (Figure 1C), only the ALC were tested in multivariate analysis. In multivariate analysis, the TNM stage (HR: 4.18, 95\%CI: 1.01-17.4, $P=0.049$,), prior or concomitant anti-VEGF targeting therapy (HR: $2.18,95 \% \mathrm{CI}: 1.15-4.14, P=0.017$ ), ECOG performance status (HR: 3.23, 95\%CI: 1.58-6.60, $P=0.001$ ), and ALC (HR: 0.38, 95\%CI: $0.23-0.62$, $P<0.001$ ), remained statistically significantly associated with OS (Table 2B). ALC were also significantly associated with OS for both, if additionally histology, age, and sex, were included in the multivariate model $(P<0.001)$ and if all other variables besides NLR and ANC were included in a multivariate model $(P=0.003)$ (Table $2 \mathrm{~B})$.

ALC and ECOG performance status remained significant in overall survival estimates adjusted for the therapy-line $(1+2$ versus $\geq 3$; Figure S3).

Contrary to our hypothesis that anti-VEGF targeting therapy may improve the clinical outcome with PD-1/PD-L1 inhibitors, prior or concomitant anti-VEGF targeting therapy was associated with a significantly inferior survival. In order to disclose differences that may explain this unexpected finding, baseline characteristics between anti-VEGF naïve and anti-VEGF exposed patients were compared. Substantial differences concerning PD-L1 positivity $(67 \%$ versus $36 \%, P=0.024)$ and concerning administration of ICB as the third line therapy and beyond $(20 \%$ versus $59 \%, P=0.002)$ were found (Table S2). 
Table 2. Univariate and multivariate analysis for PFS (A) and OS (B).

\begin{tabular}{|c|c|c|c|c|c|c|c|c|c|c|c|c|c|c|c|c|c|c|}
\hline \multirow{3}{*}{ A } & & & \multicolumn{16}{|c|}{ Progression-Free Survival } \\
\hline & & & \multicolumn{8}{|c|}{ Univariate Cox Regression Model } & \multicolumn{8}{|c|}{ Multivariate Cox Regression Model } \\
\hline & & $P^{\$}$ & $\mathrm{~N} 1$ & $\mathrm{~N} 2$ & $\mathrm{~N}$ & Events & $P$ & HR & $\begin{array}{c}\text { Lower } \\
95 \% \\
\text { CI }\end{array}$ & $\begin{array}{l}\text { Upper } \\
95 \% \\
\text { CI }\end{array}$ & $\mathrm{N}$ & Events & $P$ & HR & $\begin{array}{c}\text { Lower } \\
95 \% \\
\text { CI }\end{array}$ & $\begin{array}{l}\text { Upper } \\
95 \% \\
\text { CI }\end{array}$ & $P$ & $P$ \\
\hline Antibiotic exposure* & yes versus no & 0.080 & 62 & 80 & 142 & 109 & 0.922 & 1.02 & 0.70 & 1.49 & \multirow{20}{*}{119} & \multirow{20}{*}{91} & - & - & - & - & - & 0.047 \\
\hline Neutrophil-lymphocyte-ratio & $>3.8$ versus $\leq 3.8$ & 0.202 & 82 & 59 & 141 & 108 & 0.097 & 1.39 & 0.94 & 2.04 & & & - & - & - & - & - & - \\
\hline CNS involvement & yes versus no & 0.276 & 30 & 112 & 142 & 109 & 0.629 & 1.12 & 0.71 & 1.78 & & & - & - & - & - & - & 0.621 \\
\hline C-reactive protein $(\mathrm{mg} / \mathrm{dL})$ & $>0.6$ versus $\leq 0.6$ & 0.213 & 94 & 44 & 138 & 107 & 0.633 & 1.11 & 0.73 & 1.69 & & & - & - & - & - & - & 0.139 \\
\hline Tertiary cancer center & Linz versus Salzburg & 0.905 & 92 & 50 & 142 & 109 & 0.061 & 0.69 & 0.47 & 1.02 & & & - & - & - & - & - & 0.798 \\
\hline Histology & $\begin{array}{l}\text { squamous versus } \\
\text { non-squamous }\end{array}$ & 0.719 & 46 & 96 & 142 & 109 & 0.595 & 0.90 & 0.60 & 1.34 & & & - & - & - & - & 0.710 & 0.478 \\
\hline Sex & female versus male & 0.509 & 57 & 85 & 142 & 109 & 0.257 & 0.80 & 0.54 & 1.18 & & & - & - & - & - & 0.209 & 0.169 \\
\hline TNM stage & stage IV versus stage III & 0.541 & 127 & 15 & 142 & 109 & 0.044 & 2.03 & 1.02 & 4.03 & & & 0.476 & 1.29 & 0.64 & 2.62 & 0.415 & 0.519 \\
\hline ICB therapy line & $\geq 3$ rd line versus $<3$ rd line & 0.279 & 35 & 107 & 142 & 109 & 0.011 & 1.73 & 1.13 & 2.63 & & & 0.610 & 1.15 & 0.68 & 1.94 & 0.531 & 0.777 \\
\hline PD-L1 status & PD-L1+ versus PD-L1- & 0.212 & 75 & 44 & 119 & 91 & 0.001 & 0.49 & 0.32 & 0.75 & & & 0.053 & 0.61 & 0.37 & 1.01 & 0.050 & 0.105 \\
\hline Age & $>66$ versus $\leq 66$ years & 0.755 & 67 & 75 & 142 & 109 & 0.793 & 1.05 & 0.72 & 1.54 & & & - & - & - & - & 0.954 & 0.343 \\
\hline Smoking history & smoker versus never-smoker & 0.229 & 116 & 16 & 132 & 100 & 0.700 & 1.14 & 0.59 & 2.19 & & & - & - & - & - & - & 0.992 \\
\hline $\begin{array}{l}\text { Prior/concomitant anti-VEGF } \\
\text { therapy }\end{array}$ & yes versus no & 0.530 & 17 & 125 & 142 & 109 & 0.029 & 1.89 & 1.07 & 3.36 & & & 0.168 & 1.62 & 0.81 & 3.24 & 0.249 & 0.063 \\
\hline Prior radiotherapy & yes versus no & 0.782 & 63 & 79 & 142 & 109 & 0.670 & 0.92 & 0.63 & 1.35 & & & - & - & - & - & - & 0.341 \\
\hline $\begin{array}{c}\text { Prior/concomitant denosumab } \\
\text { therapy }\end{array}$ & yes versus no & 0.316 & 36 & 106 & 142 & 109 & 0.190 & 1.33 & 0.87 & 2.03 & & & - & - & - & - & - & 0.646 \\
\hline ECOG performance status & $>1$ versus $\leq 1$ & 0.927 & 17 & 125 & 142 & 109 & 0.001 & 2.53 & 1.49 & 4.29 & & & 0.099 & 1.84 & 0.89 & 3.77 & 0.067 & 0.141 \\
\hline EGFR mutation status & mutant versus wild-type & 0.753 & 10 & 130 & 140 & 108 & 0.083 & 1.84 & 0.92 & 3.66 & & & - & - & - & - & - & 0.192 \\
\hline ALK translocation & yes versus no & 0.239 & 3 & 131 & 134 & 103 & 0.467 & 1.53 & 0.48 & 4.86 & & & - & - & - & - & - & 0.827 \\
\hline $\begin{array}{l}\text { Absolute lymphocyte count } \\
\qquad\left(\times 10^{9} / \mathrm{L}\right)\end{array}$ & $>0.93$ versus $\leq 0.93$ & 0.796 & 100 & 41 & 141 & 108 & 0.010 & 0.58 & 0.38 & 0.88 & & & 0.012 & 0.55 & 0.35 & 0.88 & 0.030 & 0.023 \\
\hline $\begin{array}{l}\text { Absolute neutrophil count } \\
\qquad\left(\times 10^{9} / \mathrm{L}\right)\end{array}$ & $>4.83$ versus $\leq 4.83$ & 0.058 & 86 & 55 & 141 & 108 & 0.898 & 1.03 & 0.69 & 1.52 & & & - & - & - & - & - & - \\
\hline
\end{tabular}


Table 2. Cont.

\begin{tabular}{|c|c|c|c|c|c|c|c|c|c|c|c|c|c|c|c|c|c|c|}
\hline \multirow{3}{*}{ B } & & & \multicolumn{16}{|c|}{ Overall Survival } \\
\hline & & & \multicolumn{8}{|c|}{ Univariate Cox Regression Model } & \multicolumn{8}{|c|}{ Multivariate Cox Regression Models } \\
\hline & & $P^{\$}$ & $\mathrm{~N} 1$ & $\mathrm{~N} 2$ & $\mathrm{~N}$ & Events & $P$ & HR & $\begin{array}{c}\text { lower } \\
95 \% \\
\text { CI }\end{array}$ & $\begin{array}{c}\text { upper } \\
95 \% \\
\text { CI }\end{array}$ & $\mathrm{N}$ & Events & $P$ & $\mathrm{HR}$ & $\begin{array}{c}\text { Lower } \\
95 \% \\
\text { CI }\end{array}$ & $\begin{array}{c}\text { Upper } \\
95 \% \\
\text { CI }\end{array}$ & $P$ & $P$ \\
\hline Antibiotic exposure* & yes versus no & 0.117 & 62 & 80 & 142 & 76 & 0.675 & 0.90 & 0.56 & 1.45 & & & - & - & - & - & - & 0.135 \\
\hline Neutrophil-lymphocyte-ratio $^{\$}$ & $>3.8$ versus $\leq 3.8$ & 0.035 & 82 & 59 & 141 & 75 & 0.001 & 2.22 & 1.36 & 3.63 & & & - & - & - & - & - & - \\
\hline CNS involvement & yes versus no & 0.499 & 30 & 112 & 142 & 76 & 0.154 & 1.49 & 0.86 & 2.57 & & & - & - & - & - & - & 0.249 \\
\hline C-reactive protein $(\mathrm{mg} / \mathrm{dL})$ & $>0.6$ versus $\leq 0.6$ & 0.702 & 94 & 44 & 138 & 74 & 0.598 & 1.15 & 0.68 & 1.94 & & & - & - & - & - & - & 0.625 \\
\hline Tertiary cancer center & Linz versus Salzburg & 0.249 & 92 & 50 & 142 & 109 & 0.271 & 0.77 & 0.49 & 1.22 & & & - & - & - & - & - & 0.496 \\
\hline Histology & $\begin{array}{l}\text { squamous versus } \\
\text { non-squamous }\end{array}$ & 0.101 & 46 & 96 & 142 & 76 & 0.597 & 0.87 & 0.52 & 1.46 & & & - & - & - & - & 0.330 & 0.413 \\
\hline Sex & female versus male & 0.507 & 57 & 85 & 142 & 76 & 0.796 & 0.94 & 0.59 & 1.49 & & & - & - & - & - & 0.782 & 0.211 \\
\hline TNM stage & stage IV versus stage III & 0.322 & 127 & 15 & 142 & 76 & 0.018 & 5.52 & 1.35 & 22.64 & & & 0.049 & 4.18 & 1.01 & 17.36 & 0.040 & 0.126 \\
\hline ICB therapy line & $\geq 3$ rd line versus $<3$ rd line & 0.930 & 35 & 107 & 142 & 76 & 0.004 & 1.97 & 1.24 & 3.16 & 141 & 75 & 0.055 & 1.64 & 0.99 & 2.71 & 0.031 & 0.271 \\
\hline PD-L1 status & PD-L1+ versus PD-L1- & 0.757 & 75 & 44 & 119 & 61 & 0.140 & 0.68 & 0.41 & 1.13 & & & - & - & - & - & - & 0.878 \\
\hline Age & $>66$ versus $\leq 66$ years & 0.627 & 67 & 75 & 142 & 76 & 0.977 & 0.99 & 0.63 & 1.57 & & & - & - & - & - & 0.954 & 0.566 \\
\hline Smoking history & smoker versus never-smoker & 0.996 & 116 & 16 & 132 & 70 & 0.636 & 0.84 & 0.40 & 1.75 & & & - & - & - & - & - & 0.366 \\
\hline $\begin{array}{l}\text { Prior/concomitant anti-VEGF } \\
\text { therapy }\end{array}$ & yes versus no & 0.176 & 17 & 125 & 142 & 76 & 0.010 & 2.18 & 1.21 & 3.93 & & & 0.017 & 2.18 & 1.15 & 4.14 & 0.012 & 0.002 \\
\hline Prior radiotherapy & yes versus no & 0.863 & 63 & 79 & 142 & 76 & 0.579 & 1.14 & 0.72 & 1.78 & & & - & - & - & - & - & 0.284 \\
\hline $\begin{array}{c}\text { Prior/concomitant denosumab } \\
\text { therapy }\end{array}$ & yes versus no & 0.847 & 36 & 106 & 142 & 76 & 0.849 & 0.95 & 0.54 & 1.65 & & & - & - & - & - & - & 0.993 \\
\hline ECOG performance status & $>1$ versus $\leq 1$ & 0.159 & 17 & 125 & 142 & 76 & 0.007 & 2.58 & 1.30 & 5.10 & & & 0.001 & 3.23 & 1.58 & 6.60 & 0.002 & 0.887 \\
\hline EGFR mutation status & mutant versus wild-type & 0.337 & 10 & 130 & 140 & 75 & 0.100 & 1.93 & 0.88 & 4.21 & & & - & - & - & - & - & 0.585 \\
\hline ALK translocation & yes versus no & 0.365 & 3 & 131 & 134 & 71 & 0.255 & 1.96 & 0.62 & 6.27 & & & - & - & - & - & - & 0.670 \\
\hline $\begin{array}{l}\text { Absolute lymphocyte count } \\
\qquad\left(\times 10^{9} / \mathrm{L}\right)\end{array}$ & $>0.93$ versus $\leq 0.93$ & 0.136 & 100 & 41 & 141 & 75 & $<0.001$ & 0.38 & 0.24 & 0.62 & & & $<0.001$ & 0.38 & 0.23 & 0.62 & $<0.001$ & 0.003 \\
\hline $\begin{array}{l}\text { Absolute neutrophil count } \\
\qquad\left(\times 10^{9} / \mathrm{L}\right)^{\$}\end{array}$ & $>4.83$ versus $\leq 4.83$ & 0.002 & 86 & 55 & 141 & 75 & 0.207 & 1.37 & 0.84 & 2.22 & & & - & - & - & - & - & - \\
\hline
\end{tabular}

CNS: central nervous system, ICB: immune checkpoint blockade, PD-L1: programmed cell death ligand 1, VEGF: vascular endothelial growth factor, EGFR: epidermal growth factor receptor, ALK: anaplastic lymphoma kinase, HR: hazard ratio, $95 \%$ CI: $95 \%$ confidence interval. * administration of antibiotics within a time frame of month before to one month after start of immune-checkpoint blockade. ${ }^{\$}$ proportional hazard assumptions are violated, $P^{\$}: P$-values from test for proportional hazard (cox.zph). 

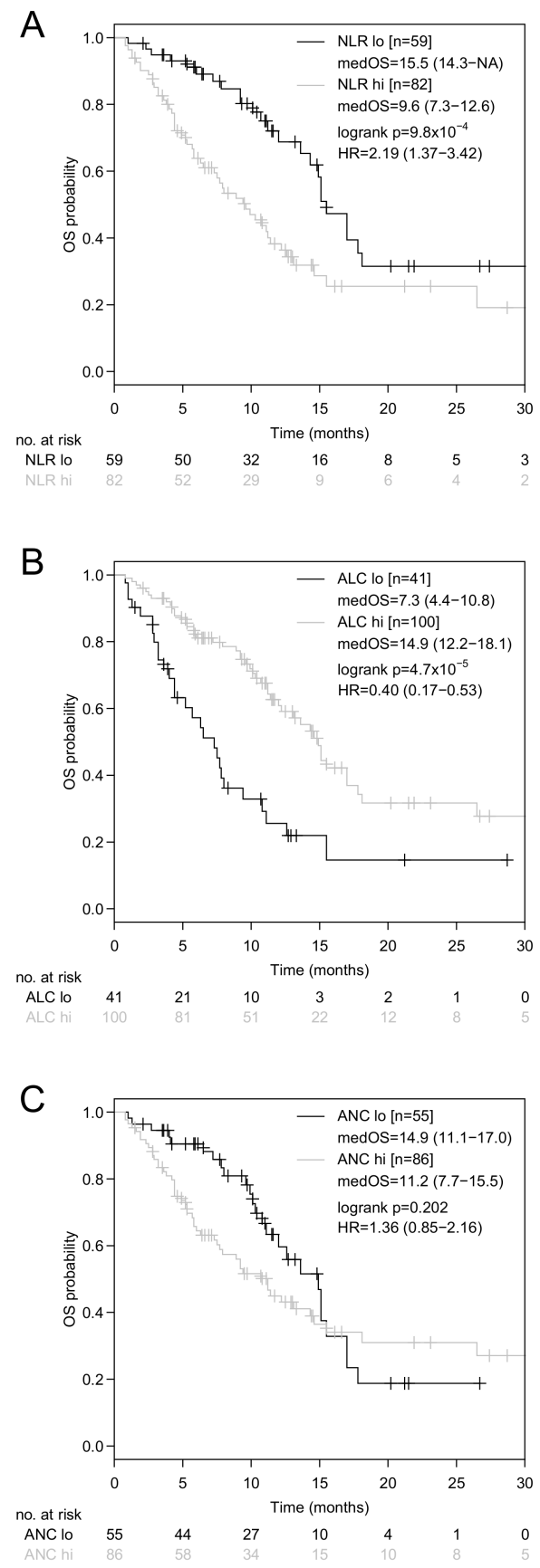

Figure 1. Kaplan-Meier curves for OS according to the baseline neutrophil-lymphocyte ratio (NLR), absolute lymphocyte count (ALC), and absolute neutrophil count (ANC). Comparison of Kaplan-Meier curves for OS in advanced NSCLC patients with a baseline NLR $>3.80$ versus $\leq 3.80$ (A), ALC $>0.93 \times 10^{9} / \mathrm{L}$ versus $\leq 0.93 \times 10^{9} / \mathrm{L}(\mathbf{B})$, and $\mathrm{ANC}>4.83 \times 10^{9} / \mathrm{L}$ versus $\leq 4.83 \times 10^{9} / \mathrm{L}(\mathbf{C})$. HR is hazard ratio, $95 \%$ confidence interval in brackets. 

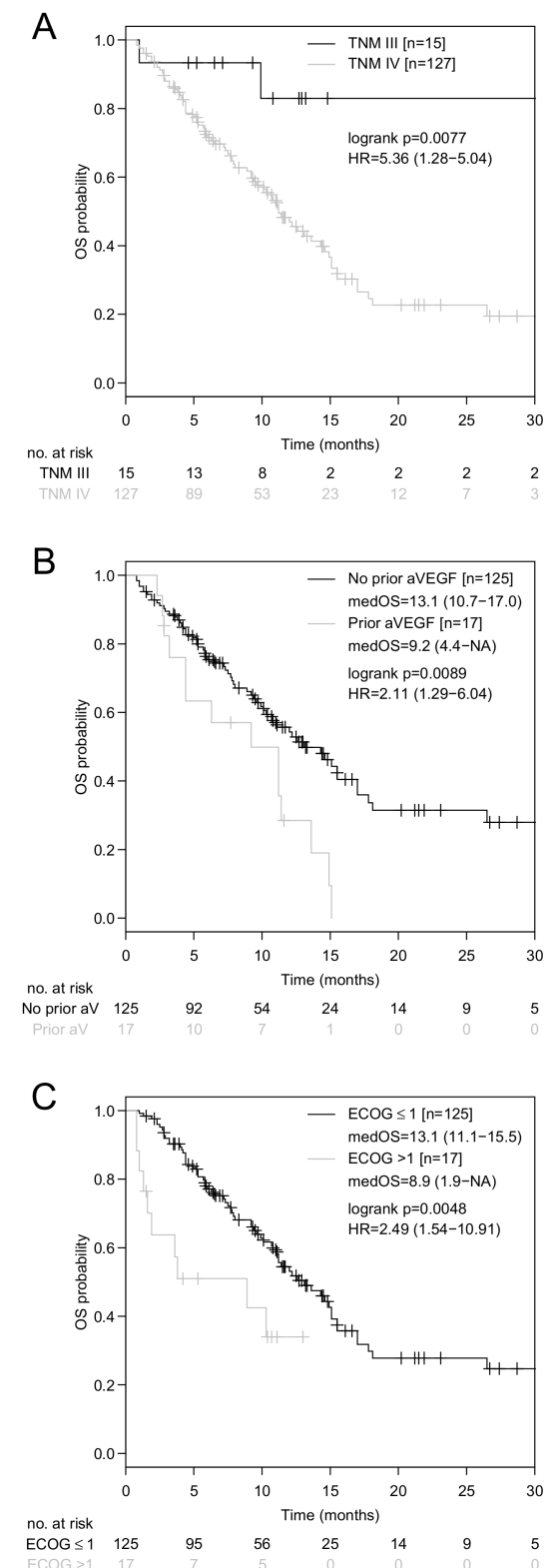

Figure 2. Kaplan-Meier curves for OS according to the TNM stage, prior or concomitant anti-VEGF targeting therapy and ECOG performance status. Comparison of Kaplan-Meier curves for OS in advanced NSCLC patients with TNM stage IV versus III (A), administration of prior or concomitant anti-VEGF targeting therapy (yes versus no) (B), and ECOG performance status $>1$ versus $\leq 1$ (C). HR is the hazard ratio with a $95 \%$ confidence interval in brackets.

\subsection{Association of ALC, ECOG Performance Status and PD-L1 Expression}

The baseline ALC and baseline ECOG performance status showed a statistically significant weak inverse correlation (Kendall tau- $\mathrm{b}=-0.17, P=0.010$, Figure 3A). Categorical PD-L1 expression was neither associated with the ECOG performance status (Kendall tau- $\mathrm{b}=-0.088, P=0.298$, Figure 3B) nor with the ALC (Kendal tau-b $=-0.007, P=0.923$, Figure 3C). 

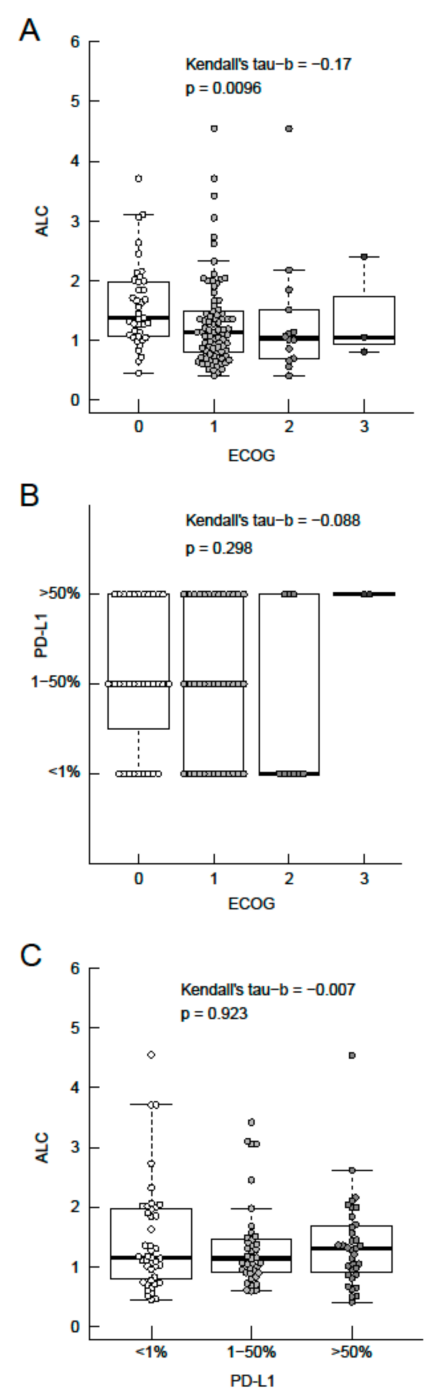

Figure 3. Association between the baseline absolute lymphocyte count (ALC), ECOG performance status, and categorical PD-L1 expression. Association between ALC and ECOG performance status (A), categorical PD-L1 expression status and ECOG performance status (B), ALC and categorical PD-L1 expression status $(\mathbf{C})$. Boxes representing the interquartile range (IQR) from the first quartile (Q1) to the third quartile (Q3) of data, thick line the median, upper whiskers the minimum of (maximum or $\mathrm{Q} 3+1.5 \times \mathrm{IQR}$ ) and lower whiskers the maximum of (minimum or Q1-1.5 $\times$ IQR).3.4. Association of Antibiotic Exposure and Survival with Immune-Checkpoint Blockade.

Concomitant application of antibiotics with initiation of PD-1/PD-L1 blockade did neither affect median PFS ( $\mathrm{AB}^{+}: 3.8$ months (95\%CI: 2.5-7.4) versus $\mathrm{AB}^{-}: 4.0$ months (95\%CI: 3.1-5.7), HR: 1.02 (95\%CI: 0.69-1.50), $P=0.920$, Figure S4A) nor median OS $\left(\mathrm{AB}^{+}: 14.6\right.$ months $(95 \% \mathrm{CI}: 9.4-\mathrm{NA})$ versus $\mathrm{AB}^{-}: 11.2$ months (95\%CI: 9.9-15.1), HR: 0.91 (95\%CI: 0.57-1.45), $P=0.675$, Figure $44 \mathrm{~B}$ ) in the entire cohort.

\section{Discussion}

In this retrospective analysis, the TNM stage (IV versus III, HR 4.18), prior anti-VEGF targeting therapy (yes versus no, HR: 2.18), ECOG performance status (ECOG > 1 versus ECOG $\leq 1$, HR: 3.23), and ALC (high: $>0.93 \times 10^{9} / \mathrm{L}$ versus $\leq 0.93 \times 10^{9} / \mathrm{L}$, HR: 0.38 ) were identified as the most relevant baseline parameters associated with OS in aNSCLC treated with ICB (Table 2B). We are convinced that 
assessment of baseline ECOG performance status and ALC before the initiation of ICB is routinely carried out and, thus, feasible for clinical use in any lung cancer unit.

Subgroup analyses of the vast majority of trials showed that a better baseline ECOG performance status is associated with a superior clinical outcome in patients with aNSCLC undergoing palliative immune-checkpoint inhibitor therapy $[5,6,8-10,12]$, chemotherapy [47-49], or targeted therapy [50,51]. Considering the impact of the ECOG performance status on OS irrespective of the type of systemic therapy, this parameter is more likely to be of a prognostic value than of a predictive value. Due to the fact that, in the general inclusion of NSCLC patients in immune-checkpoint inhibitor trials is restricted to an ECOG performance status $\leq 1$ [5,8-10,12], the actual impact of the ECOG performance status on OS is not depicted in these studies.

As a surrogate marker for systemic inflammation, the baseline NLR at initiation of ICB was significantly associated with OS in our NSCLC cohort. In general, the favorable outcome of ICB in patients with a low baseline NLR does not come at the cost of increased toxicity [52]. Due to the retrospective nature of this study, assessment of immune-related adverse events was not performed. However, in our NSCLC cohort, the impact of the NLR on OS (Figure 1A) was predominantly driven by variations in the ALC (Figure 1B), while variations in the ANC played a subsidiary role (Figure 1C). The calculated ALC cut-off in our study closely resembles the lower limit of normal and is, therefore, suitable for use in clinical practice. Baseline CRP levels above the upper limit of normal $(>0.6 \mathrm{mg} / \mathrm{dL})$ did neither affect PFS nor OS (Table 2), which was also the case for higher CRP cut-off values ( $>5 \mathrm{mg} / \mathrm{dL}$ versus $\leq 5 \mathrm{mg} / \mathrm{dL}$ ). Although ECOG performance status and ALC proved to be independently associated with OS in multivariate analysis, a highly significant inverse correlation was found between these two parameters (Figure 3A). The latter finding is in line with previous reports [53].

In contrast to the majority of phase III trial data [6-12], in our NSCLC cohort, the PD-L1 expression on tumor cells was not associated with OS (Figure S2B). It is noteworthy that $73 \%$ of patients received ICB as second or later-line therapy in our cohort. Due to prior systemic therapy application and due to the fact that PD-L1 expression is a dynamic parameter, the PD-L1 expression reported in the initial diagnostic biopsy may not reflect the actual PD-L1 expression at the time point of ICB initiation [16]. Central assessment of the PD-L1 expression status for both oncologic centers was carried out by a single experienced lung pathologist and, therefore, staining results could not be biased by inter-observer variations.

Antibiotic exposure in temporal proximity to the initiation of ICB did neither impact PFS (Figure S4A) nor OS (Figure S4B) in our bicentric NSCLC cohort. Published data on the influence of antibiotics on clinical outcome are conflicting [54]. While several retrospective studies reported a detrimental effect of antibiotic exposure in temporal proximity to the start of ICB in aNSCLC [18,20,22], including our single-center experience with non-squamous NSCLC patients [19], Metges et al. reported a survival advantage for NSCLC patients receiving antibiotics prior to the immune-checkpoint inhibitor therapy [55]. In consideration of the limited data and the conflicting results, a prospective evaluation of the impact of antibiotics on clinical outcome with immune-checkpoint inhibition is necessary in future clinical ICB trials.

In our cohort, one out of four patients received denosumab prior to and/or concomitantly with ICB. Anti-RANKL treatment has been shown to protect T-cells specific for melanoma antigens in transgenic mouse models by depletion of medullary thymic epithelial cells, which interferes with a negative selection. Blockade of the RANK-RANKL interaction resulted in improved survival in mice, which had been previously inoculated with melanoma cells [56]. In an animal model, Ahern et al. found that RANK was mainly expressed by tumor-infiltrating lymphocytes while RANKL expression was largely restricted to tumor-infiltrating macrophages, dendritic cells, and myeloid-derived suppressor cells. The co-administration of RANKL-targeting antibodies and immune-checkpoint inhibitors increased intra-tumoral density of CD8 ${ }^{+}$T-cells and enhanced antitumor activity [44,45]. However, denosumab co-administration did not impact the clinical outcome in our analysis (Table 2). 
Anti-VEGF targeting therapy in addition to chemotherapy increased the number of $\mathrm{CD}^{+}$ T-cells in the peripheral blood in advanced melanoma patients [57] and improved antigen-specific $\mathrm{CD}^{+}{ }^{+} \mathrm{T}$-cell responses in vivo and in vitro in aNSCLC [40]. In animal models, interferon-gamma induced upregulation of PD-L1 drove secondary resistance to VEGF-blockade while concurrent anti-angiogenic therapy and PD-L1 inhibition induced formation of high endothelial venules, which facilitated T-cell infiltration and enhanced anti-tumor activity [58]. Seventeen patients (12\%) had been treated with anti-VEGF targeting therapy prior to the initiation of immune-checkpoint inhibition or concomitantly with PD-1/PD-L1 blockade in our NSCLC cohort. Prior or concomitant anti-VEGF blockade was associated with inferior OS (Figure 2B, Table 2B). The latter finding may be explained by immune-checkpoint inhibition being applied in later therapy lines in anti-VEGF exposed patients when compared to anti-VEGF naïve patients (Table S2). In the IMpower150 study, Socinski et al. investigated carboplatin plus paclitaxel and bevacizumab with and without atezolizumab as front-line systemic therapy in non-squamous aNSCLC and found an OS benefit of 4.5 months with the addition of ICB [12]. However, the report on clinical outcome of patients treated with carboplatin, paclitaxel, and atezolizumab without bevacizumab is eagerly awaited to clarify the role of concomitant anti-VEGF blockade in this setting.

\section{Conclusions}

In conclusion, the results of our analysis demonstrate the practicality of estimating OS from initiating the PD-1/PD-L1 blockade based on baseline ALC and ECOG performance status in aNSCLC. A high baseline ALC was associated with a good ECOG performance status. Stratification according to ALC and ECOG status in future clinical NSCLC trials investigating ICB is warranted. Neither PD-L1 expression status, nor antibiotic treatment status, had an impact on OS in our NSCLC cohort. The question arises whether the negative impact of antibiotic exposure on OS in previous reports is caused by interference with ICB or is caused by the underlying condition necessitating antibiotic administration. The negative impact of anti-VEGF targeting therapy preceding immune-checkpoint inhibitor therapy was unexpected but may be explained by administration of PD-1/PD-L1 inhibitors in later therapy-lines. Further data from the IMpower150 study may eventually clarify the role of anti-VEGF therapy in combination with ICB in aNSCLC.

Supplementary Materials: The following are available online at http://www.mdpi.com/2077-0383/8/7/1014/s1, Figure S1: Kaplan-Meier curves for PFS (A) and OS (B) from initiation of PD-1/PD-L1 blockade in 142 advanced NSCLC patients. Figure S2: Kaplan-Meier curves for PFS and OS according to PD-L1 expression status on tumor cells. Figure S3: Therapy line adjusted survival curves for PFS and OS according to absolute lymphocyte count and ECOG performance status. Figure S4: Kaplan-Meier curves for PFS and OS according to antibiotic treatment status. Table S1: Optimal quantile cut-offs for dichotomization of patients for NLR, ALC, and ANC based on maximal Harrel's C-index. Table S2: Comparison of baseline characteristics between anti-VEGF exposed and anti-VEGF naïve advanced NSCLC patients receiving PD-1/PD-L1 blockade.

Author Contributions: Conceptualization: F.H., G.R., T.W., and R.G. Methodology: F.H., G.R., T.W., L.W., S.G., G.H., R.G., D.L., and B.L. Software: F.H., G.R., and H.H. Validation: F.H., G.R., and H.H. Formal Analysis: F.H., G.R., S.G., G.H., and H.H. Investigation: F.H. and G.R. Resources: F.H., G.R., T.W., S.G., and L.W. Data Curation: F.H, G.R., and H.H. Writing - Original Draft Preparation: F.H., G.R., and R.G. Writing-Review \& Editing: F.H., T.W., D.L., S.G., G.H., H.H., G.R., B.L., L.W., and R.G. Visualization: F.H., G.R., and H.H. Supervision: G.R. and R.G. Project Administration: F.H. and G.R.

Conflicts of Interest: F.H. received travel grants from Bristol-Myers Squibb, Roche, and Merck Sharp \& Dohme. D.L. received travel grants from Roche. T.W. received travel grants from Roche. S.P.G. received speakers' honoraria from Bristol-Myers Squibb and Roche, travel grants from Merck and Roche, and research funding from Roche. G.H. has acted as scientific advisor for Bristol-Myers Squibb, Roche, and Merck Sharp \& Dohme. L.W. received research support from Roche, speakers' honoraria from Merck and Bristol-Myers Squibb. H.H. declares no conflicts of interest. B.L. has acted as scientific advisor for, or obtained speakers' honoraria from Bristol-Myers Squibb, Merck Sharp \& Dohme, and Roche. G.R. has acted as scientific advisor for Roche, obtained speakers' honoraria from Bristol-Myers Squibb and Roche, received travel grants from Roche, and received research funding from Roche. R.G. received speakers' honoraria from Roche, Merck/Merck Sharp \& Dohme and Bristol-Myers Squibb, has acted as a scientific advisor for Roche and Bristol-Myers Squibb, received research funding from Roche, Merck/Merck Sharp \& Dohme, and Bristol-Myers Squibb, and obtained travel grants from Roche. 


\section{References}

1. Siegel, R.L.; Miller, K.D.; Jemal, A. Cancer Statistics, 2017. CA Cancer J. Clin. 2017, 67, 7-30. [CrossRef] [PubMed]

2. Malvezzi, M.; Carioli, G.; Bertuccio, P.; Rosso, T.; Boffetta, P.; Levi, F.; La Vecchia, C.; Negri, E. European cancer mortality predictions for the year 2016 with focus on leukaemias. Ann. Oncol. Off. J. Eur. Soc. Med. Oncol. 2016, 27, 725-731. [CrossRef]

3. Vokes, E.E.; Ready, N.; Felip, E.; Horn, L.; Burgio, M.A.; Antonia, S.J.; Aren Frontera, O.; Gettinger, S.; Holgado, E.; Spigel, D.; et al. Nivolumab versus docetaxel in previously treated advanced non-small-cell lung cancer (CheckMate 017 and CheckMate 057): 3-Year update and outcomes in patients with liver metastases. Ann. Oncol. Off. J. Eur. Soc. Med. Oncol. 2018, 29, 959-965. [CrossRef] [PubMed]

4. Felip, E.; Hellmann, M.D.; Hui, R.; Carcereny, E.; Leighl, N.B.; Ahn, M.J.; Eder, J.P.; Balmanoukian, A.S.; Aggarwal, C.; Horn, L.; et al. 4-year overall survival for patients with advanced NSCLC treated with pembrolizumab: Results from KEYNOTE-001. J. Clin. Oncol. 2018, 36 (Suppl. Abstr. 9030).

5. Brahmer, J.; Reckamp, K.L.; Baas, P.; Crino, L.; Eberhardt, W.E.; Poddubskaya, E.; Antonia, S.; Pluzanski, A.; Vokes, E.E.; Holgado, E.; et al. Nivolumab versus Docetaxel in Advanced Squamous-Cell Non-Small-Cell Lung Cancer. N. Engl. J. Med. 2015, 373, 123-135. [CrossRef] [PubMed]

6. Borghaei, H.; Paz-Ares, L.; Horn, L.; Spigel, D.R.; Steins, M.; Ready, N.E.; Chow, L.Q.; Vokes, E.E.; Felip, E.; Holgado, E.; et al. Nivolumab versus Docetaxel in Advanced Nonsquamous Non-Small-Cell Lung Cancer. N. Engl. J. Med. 2015, 373, 1627-1639. [CrossRef] [PubMed]

7. Herbst, R.S.; Baas, P.; Kim, D.W.; Felip, E.; Perez-Gracia, J.L.; Han, J.Y.; Molina, J.; Kim, J.H.; Arvis, C.D.; Ahn, M.J.; et al. Pembrolizumab versus docetaxel for previously treated, PD-L1-positive, advanced non-small-cell lung cancer (KEYNOTE-010): A randomised controlled trial. Lancet 2016, 387, 1540-1550. [CrossRef]

8. Reck, M.; Rodriguez-Abreu, D.; Robinson, A.G.; Hui, R.; Csoszi, T.; Fulop, A.; Gottfried, M.; Peled, N.; Tafreshi, A.; Cuffe, S.; et al. Pembrolizumab versus Chemotherapy for PD-L1-Positive Non-Small-Cell Lung Cancer. N. Engl. J. Med. 2016, 375, 1823-1833. [CrossRef]

9. Gandhi, L.; Rodriguez-Abreu, D.; Gadgeel, S.; Esteban, E.; Felip, E.; De Angelis, F.; Domine, M.; Clingan, P.; Hochmair, M.J.; Powell, S.F.; et al. Pembrolizumab plus Chemotherapy in Metastatic Non-Small-Cell Lung Cancer. N. Engl. J. Med. 2018, 378, 2078-2092. [CrossRef]

10. Paz-Ares, L.; Luft, A.; Vicente, D.; Tafreshi, A.; Gumus, M.; Mazieres, J.; Hermes, B.; Cay Senler, F.; Csoszi, T.; Fulop, A. Pembrolizumab plus Chemotherapy for Squamous Non-Small-Cell Lung Cancer. N. Engl. J. Med. 2018. [CrossRef]

11. Rittmeyer, A.; Barlesi, F.; Waterkamp, D.; Park, K.; Ciardiello, F.; von Pawel, J.; Gadgeel, S.M.; Hida, T.; Kowalski, D.M.; Dols, M.C.; et al. Atezolizumab versus docetaxel in patients with previously treated non-small-cell lung cancer (OAK): A phase 3, open-label, multicentre randomised controlled trial. Lancet 2017, 389, 255-265. [CrossRef]

12. Socinski, M.A.; Jotte, R.M.; Cappuzzo, F.; Orlandi, F.; Stroyakovskiy, D.; Nogami, N.; Rodriguez-Abreu, D.; Moro-Sibilot, D.; Thomas, C.A.; Barlesi, F.; et al. Atezolizumab for First-Line Treatment of Metastatic Nonsquamous NSCLC. N. Engl. J. Med. 2018, 378, 2288-2301. [CrossRef] [PubMed]

13. Antonia, S.J.; Villegas, A.; Daniel, D.; Vicente, D.; Murakami, S.; Hui, R.; Kurata, T.; Chiappori, A.; Lee, K.H.; de Wit, M.; et al. Overall Survival with Durvalumab after Chemoradiotherapy in Stage III NSCLC. N. Engl. J. Med. 2018. [CrossRef]

14. Hellmann, M.D.; Ciuleanu, T.E.; Pluzanski, A.; Lee, J.S.; Otterson, G.A.; Audigier-Valette, C.; Minenza, E.; Linardou, H.; Burgers, S.; Salman, P.; et al. Nivolumab plus Ipilimumab in Lung Cancer with a High Tumor Mutational Burden. N. Engl. J. Med. 2018, 378, 2093-2104. [CrossRef] [PubMed]

15. Jotte, R.M.; Cappuzzo, F.; Vynnychenko, I.; Stroyakovskiy, D.; Abreu, D.R.; Hussein, M.A.; Soo, R.A.; Conter, H.J.; Kozuki, T.; Silva, C.; et al. IMpower131: Primary PFS and safety analysis of a randomized phase III study of atezolizumab + carboplatin + paclitaxel or nab-paclitaxel vs carboplatin + nab-paclitaxel as 1L therapy in advanced squamous NSCLC. J. Clin. Oncol. 2018, 36 (Suppl. Abstr. LBA9000).

16. Yu, H.; Boyle, T.A.; Zhou, C.; Rimm, D.L.; Hirsch, F.R. PD-L1 Expression in Lung Cancer. J. Thorac. Oncol. 2016, 11, 964-975. [CrossRef] [PubMed] 
17. Gridelli, C.; Ardizzoni, A.; Barberis, M.; Cappuzzo, F.; Casaluce, F.; Danesi, R.; Troncone, G.; De Marinis, F. Predictive biomarkers of immunotherapy for non-small cell lung cancer: Results from an Experts Panel Meeting of the Italian Association of Thoracic Oncology. Transl. Lung. Cancer. Res. 2017, 6, 373-386. [CrossRef]

18. Routy, B.; Le Chatelier, E.; Derosa, L.; Duong, C.P.M.; Alou, M.T.; Daillere, R.; Fluckiger, A.; Messaoudene, M.; Rauber, C.; Roberti, M.P.; et al. Gut microbiome influences efficacy of PD-1-based immunotherapy against epithelial tumors. Science 2017. [CrossRef]

19. Huemer, F.; Rinnerthaler, G.; Westphal, T.; Hackl, H.; Hutarew, G.; Gampenrieder, S.P.; Weiss, L.; Greil, R. Impact of antibiotic treatment on immune-checkpoint blockade efficacy in advanced non-squamous non-small cell lung cancer. Oncotarget 2018, 9, 16512-16520. [CrossRef]

20. Derosa, L.; Hellmann, M.D.; Spaziano, M.; Halpenny, D.; Fidelle, M.; Rizvi, H.; Long, N.; Plodkowski, A.J.; Arbour, K.C.; Chaft, J.E.; et al. Negative association of antibiotics on clinical activity of immune checkpoint inhibitors in patients with advanced renal cell and non-small-cell lung cancer. Ann. Oncol. Off. J. Eur. Soc. Med. Oncol. 2018, 29, 1437-1444. [CrossRef]

21. Tinsley, N.; Zhou, C.; Villa, S.; Tan, G.; Lorigan, P.; Blackhall, F.H.; Elliott, T.; Krebs, M.; Carter, L.; Thistlethwaite, F.; et al. Cumulative antibiotic use and efficacy of immune checkpoint inhibitors in patients with advanced cancer. J. Clin. Oncol. 2018, 36 (Suppl. Abstr. 3010). [CrossRef]

22. Ahmed, J.; Kumar, A.; Parikh, K.; Anwar, A.; Knoll, B.M.; Puccio, C.; Chun, H.; Fanucchi, M.; Lim, S.H. Use of broad-spectrum antibiotics impacts outcome in patients treated with immune checkpoint inhibitors. Oncoimmunology 2018.

23. Yarchoan, M.; Hopkins, A.; Jaffee, E.M. Tumor Mutational Burden and Response Rate to PD-1 Inhibition. N. Engl. J. Med. 2017, 377, 2500-2501. [CrossRef] [PubMed]

24. Schumacher, T.N.; Schreiber, R.D. Neoantigens in cancer immunotherapy. Science 2015, 348, 69-74. [CrossRef] [PubMed]

25. Yi, M.; Qin, S.; Zhao, W.; Yu, S.; Chu, Q.; Wu, K. The role of neoantigen in immune checkpoint blockade therapy. Exp. Hematol. Oncol. 2018, 7, 28. [CrossRef] [PubMed]

26. Ramalingam, S.S.; Hellmann, M.D.; Awad, M.M.; Borghaei, H.; Gainor, J.; Brahmer, J.; Spigel, D.; Reck, M.; O’Byrne, K.J.; Paz-Ares, L.; et al. Tumor mutational burden (TMB) as a biomarker for clinical benefit from dual immune checkpoint blockade with nivolumab (nivo) + ipilimumab (ipi) in first-line (1L) non-small cell lung cancer (NSCLC): Identification of TMB cutoff from CheckMate 568. Presented at the American Association for Cancer Research 2018 Annual Meeting, Chicago, IL, USA, 14-18 April 2018.

27. Lisberg, A.; Garon, E.B. The Italian Nivolumab Expanded Access Program Confirms the Limitations of Single-Agent PD-1 Inhibition in EGFR-Mutant and Never-Smoking Patients with NSCLC. J. Thorac. Oncol. 2018, 13, 1058-1059. [CrossRef] [PubMed]

28. Lee, C.K.; Man, J.; Lord, S.; Links, M.; Gebski, V.; Mok, T.; Yang, J.C. Checkpoint Inhibitors in Metastatic EGFR-Mutated Non-Small Cell Lung Cancer-A Meta-Analysis. J. Thorac. Oncol. 2017, 12, 403-407. [CrossRef] [PubMed]

29. Mantovani, A.; Allavena, P.; Sica, A.; Balkwill, F. Cancer-related inflammation. Nature 2008, 454, $436-444$. [CrossRef]

30. Coussens, L.M.; Werb, Z. Inflammation and cancer. Nature 2002, 420, 860-867. [CrossRef]

31. Forrest, L.M.; McMillan, D.C.; McArdle, C.S.; Angerson, W.J.; Dunlop, D.J. Evaluation of cumulative prognostic scores based on the systemic inflammatory response in patients with inoperable non-small-cell lung cancer. Br. J. Cancer 2003, 89, 1028-1030. [CrossRef]

32. Guthrie, G.J.; Charles, K.A.; Roxburgh, C.S.; Horgan, P.G.; McMillan, D.C.; Clarke, S.J. The systemic inflammation-based neutrophil-lymphocyte ratio: Experience in patients with cancer. Crit. Rev. Oncol. Hematol. 2013, 88, 218-230. [CrossRef] [PubMed]

33. Templeton, A.J.; McNamara, M.G.; Seruga, B.; Vera-Badillo, F.E.; Aneja, P.; Ocana, A.; Leibowitz-Amit, R.; Sonpavde, G.; Knox, J.J.; Tran, B.; et al. Prognostic role of neutrophil-to-lymphocyte ratio in solid tumors: A systematic review and meta-analysis. J. Natl. Cancer Inst. 2014, 106, dju124. [CrossRef] [PubMed]

34. Diem, S.; Schmid, S.; Krapf, M.; Flatz, L.; Born, D.; Jochum, W.; Templeton, A.J.; Fruh, M. Neutrophil-to-Lymphocyte ratio (NLR) and Platelet-to-Lymphocyte ratio (PLR) as prognostic markers in patients with non-small cell lung cancer (NSCLC) treated with nivolumab. Lung Cancer 2017, 111, $176-181$. [CrossRef] [PubMed] 
35. Bagley, S.J.; Kothari, S.; Aggarwal, C.; Bauml, J.M.; Alley, E.W.; Evans, T.L.; Kosteva, J.A.; Ciunci, C.A.; Gabriel, P.E.; Thompson, J.C.; et al. Pretreatment neutrophil-to-lymphocyte ratio as a marker of outcomes in nivolumab-treated patients with advanced non-small-cell lung cancer. Lung Cancer 2017, 106, 1-7. [CrossRef] [PubMed]

36. Bumma, N.; Jeyakumar, G.; Kim, S.; Galasso, C.; Thakur, M.K.; Gadgeel, S.M.; Vaishampayan, U.N.; Wozniak, A.J. Neutrophil lymphocyte ratio (NLR) as a predictive biomarker for PD-1/PD-L1 directed therapy in metastatic non-small cell lung cancer (NSCLC). J. Clin. Oncol. 2017, 35, e20633. [CrossRef]

37. Tanizaki, J.; Haratani, K.; Hayashi, H.; Chiba, Y.; Nakamura, Y.; Yonesaka, K.; Kudo, K.; Kaneda, H.; Hasegawa, Y.; Tanaka, K.; et al. Peripheral Blood Biomarkers Associated with Clinical Outcome in Non-Small Cell Lung Cancer Patients Treated with Nivolumab. J. Thorac. Oncol. 2018, 13, 97-105. [CrossRef] [PubMed]

38. Sandler, A.; Gray, R.; Perry, M.C.; Brahmer, J.; Schiller, J.H.; Dowlati, A.; Lilenbaum, R.; Johnson, D.H. Paclitaxel-carboplatin alone or with bevacizumab for non-small-cell lung cancer. N. Engl. J. Med. 2006, 355, 2542-2550. [CrossRef]

39. National Comprehensive Cancer Network. Non-Small Cell Lung Cancer (Version 5.2019). Available online: https://www.nccn.org/professionals/physician_gls/pdf/nscl.pdf (accessed on 3 July 2019).

40. Martino, E.C.; Misso, G.; Pastina, P.; Costantini, S.; Vanni, F.; Gandolfo, C.; Botta, C.; Capone, F.; Lombardi, A.; Pirtoli, L.; et al. Immune-modulating effects of bevacizumab in metastatic non-small-cell lung cancer patients. Cell Death Discov. 2016, 2, 16025. [CrossRef]

41. Dougall, W.C.; Glaccum, M.; Charrier, K.; Rohrbach, K.; Brasel, K.; De Smedt, T.; Daro, E.; Smith, J.; Tometsko, M.E.; Maliszewski, C.R.; et al. RANK is essential for osteoclast and lymph node development. Genes Dev. 1999, 13, 2412-2424.

42. Henry, D.H.; Costa, L.; Goldwasser, F.; Hirsh, V.; Hungria, V.; Prausova, J.; Scagliotti, G.V.; Sleeboom, H.; Spencer, A.; Vadhan-Raj, S.; et al. Randomized, double-blind study of denosumab versus zoledronic acid in the treatment of bone metastases in patients with advanced cancer (excluding breast and prostate cancer) or multiple myeloma. J. Clin. Oncol. Off. J. Am. Soc. Clin. Oncol. 2011, 29, 1125-1132. [CrossRef]

43. Scagliotti, G.V.; Hirsh, V.; Siena, S.; Henry, D.H.; Woll, P.J.; Manegold, C.; Solal-Celigny, P.; Rodriguez, G.; Krzakowski, M.; Mehta, N.D.; et al. Overall survival improvement in patients with lung cancer and bone metastases treated with denosumab versus zoledronic acid: Subgroup analysis from a randomized phase 3 study. J. Thorac. Oncol. 2012, 7, 1823-1829. [CrossRef] [PubMed]

44. Ahern, E.; Harjunpaa, H.; Barkauskas, D.; Allen, S.; Takeda, K.; Yagita, H.; Wyld, D.; Dougall, W.C.; Teng, M.W.L.; Smyth, M.J. Co-administration of RANKL and CTLA4 Antibodies Enhances Lymphocyte-Mediated Antitumor Immunity in Mice. Clin. Cancer Res. 2017, 23, 5789-5801. [CrossRef] [PubMed]

45. Ahern, E.; Harjunpaa, H.; O’Donnell, J.S.; Allen, S.; Dougall, W.C.; Teng, M.W.L.; Smyth, M.J. RANKL blockade improves efficacy of PD1-PD-L1 blockade or dual PD1-PD-L1 and CTLA4 blockade in mouse models of cancer. Oncoimmunology 2018, 7, e1431088. [CrossRef] [PubMed]

46. Ettinger, D.S.; Aisner, D.L.; Wood, D.E.; Akerley, W.; Bauman, J.; Chang, J.Y.; Chirieac, L.R.; D'Amico, T.A.; Dilling, T.J.; Dobelbower, M.; et al. NCCN Guidelines Insights: Non-Small Cell Lung Cancer, Version 5.2018. J. Natl. Compr. Canc. Netw. 2018, 16, 807-821. [CrossRef] [PubMed]

47. Paz-Ares, L.G.; de Marinis, F.; Dediu, M.; Thomas, M.; Pujol, J.L.; Bidoli, P.; Molinier, O.; Sahoo, T.P.; Laack, E.; Reck, M.; et al. PARAMOUNT: Final overall survival results of the phase III study of maintenance pemetrexed versus placebo immediately after induction treatment with pemetrexed plus cisplatin for advanced nonsquamous non-small-cell lung cancer. J. Clinic. Oncol. Off. J. Am. Soc. Clinic. Oncol. 2013, 31, 2895-2902. [CrossRef]

48. Georgoulias, V.; Ardavanis, A.; Agelidou, A.; Agelidou, M.; Chandrinos, V.; Tsaroucha, E.; Toumbis, M.; Kouroussis, C.; Syrigos, K.; Polyzos, A.; et al. Docetaxel versus docetaxel plus cisplatin as front-line treatment of patients with advanced non-small-cell lung cancer: A randomized, multicenter phase III trial. J. Clin. Oncol. Off. J. Am. Soc. Clin. Oncol. 2004, 22, 2602-2609. [CrossRef]

49. Scagliotti, G.V.; Parikh, P.; von Pawel, J.; Biesma, B.; Vansteenkiste, J.; Manegold, C.; Serwatowski, P.; Gatzemeier, U.; Digumarti, R.; Zukin, M.; et al. Phase III study comparing cisplatin plus gemcitabine with cisplatin plus pemetrexed in chemotherapy-naive patients with advanced-stage non-small-cell lung cancer. J. Clin. Oncol. Off. J. Am. Soc. Clin. Oncol. 2008, 26, 3543-3551. [CrossRef] 
50. Sequist, L.V.; Yang, J.C.; Yamamoto, N.; O’Byrne, K.; Hirsh, V.; Mok, T.; Geater, S.L.; Orlov, S.; Tsai, C.M.; Boyer, M.; et al. Phase III study of afatinib or cisplatin plus pemetrexed in patients with metastatic lung adenocarcinoma with EGFR mutations. J. Clin. Oncol. Off. J. Am. Soc. Clin. Oncol. 2013, 31, 3327-3334. [CrossRef]

51. Soria, J.C.; Ohe, Y.; Vansteenkiste, J.; Reungwetwattana, T.; Chewaskulyong, B.; Lee, K.H.; Dechaphunkul, A.; Imamura, F.; Nogami, N.; Kurata, T.; et al. Osimertinib in Untreated EGFR-Mutated Advanced Non-Small-Cell Lung Cancer. N. Engl. J. Med. 2018, 378, 113-125. [CrossRef]

52. Khoja, L.; Atenafu, E.G.; Templeton, A.; Qye, Y.; Chappell, M.A.; Saibil, S.; Hogg, D.; Butler, M.O.; Joshua, A.M. The full blood count as a biomarker of outcome and toxicity in ipilimumab-treated cutaneous metastatic melanoma. Cancer Med. 2016, 5, 2792-2799. [CrossRef]

53. Kacan, T.; Babacan, N.A.; Seker, M.; Yucel, B.; Bahceci, A.; Eren, A.A.; Eren, M.F.; Kilickap, S. Could the neutrophil to lymphocyte ratio be a poor prognostic factor for non small cell lung cancers? Asian Pac. J. Cancer Prev. 2014, 15, 2089-2094. [CrossRef] [PubMed]

54. Huemer, F.; Rinnerthaler, G.; Lang, D.; Hackl, H.; Lamprecht, B.; Greil, R. Association between antibiotics use and outcome in patients with NSCLC treated with immunotherapeutics. Ann. Oncol. Off. J. Eur. Soc. Med. Oncol. 2019. [CrossRef] [PubMed]

55. Metges, J.; Michaud, E.; Lagadec, D.D.; Marhuenda, F.; Chaslerie, A.; Grude, F. Impact of anti-infectious and corticosteroids on immunotherapy: Nivolumab and Pembrolizumab follow-up in a French study. Ann. Oncol. 2018, 29 (Suppl. 8), viii400-viii441. [CrossRef]

56. Khan, I.S.; Mouchess, M.L.; Zhu, M.L.; Conley, B.; Fasano, K.J.; Hou, Y.; Fong, L.; Su, M.A.; Anderson, M.S. Enhancement of an anti-tumor immune response by transient blockade of central T cell tolerance. J. Exp. Med. 2014, 211, 761-768. [CrossRef] [PubMed]

57. Mansfield, A.S.; Nevala, W.K.; Lieser, E.A.; Leontovich, A.A.; Markovic, S.N. The immunomodulatory effects of bevacizumab on systemic immunity in patients with metastatic melanoma. Oncoimmunology 2013, 2, e24436. [CrossRef]

58. Allen, E.; Jabouille, A.; Rivera, L.B.; Lodewijckx, I.; Missiaen, R.; Steri, V.; Feyen, K.; Tawney, J.; Hanahan, D.; Michael, I.P.; et al. Combined antiangiogenic and anti-PD-L1 therapy stimulates tumor immunity through HEV formation. Sci. Transl. Med. 2017, 9. [CrossRef] 